\title{
The Structural Relationship Among Perceived Positive and Negative Parenting Attitude, Life Skills, and Transfer of Chinese Student-Athletes
}

\section{Qi Yi Wang}

Yong In University

Taehee Lim

Yong In University

Junsu Bae ( $\sim$ junsubae57@naver.com )

Yong In University

\section{Research Article}

Keywords: positive youth development, youth sport, parental behavior, mediation effect

Posted Date: October 8th, 2021

DOI: https://doi.org/10.21203/rs.3.rs-952083/v1

License: (c) (1) This work is licensed under a Creative Commons Attribution 4.0 International License.

Read Full License 


\section{Abstract}

A parenting attitude is regarded as a very important factor influencing the positive development of student-athletes. However, there are far fewer studies on parenting attitude in sport PYD area. This study aimed to understand the relationship among the positive/negative parenting attitudes, life skills, and transfer. Participants were 257 Chinese student-athletes (male=171, female=86, Mage=15.70) in track and field, basketball, soccer, and taekwondo. Date were analyzed by using descriptive statistic, correlation, and Structural Equation Modeling (SEM). SEM analysis showed that parenting attitude was significantly related to life skills development and transfer. A positive parenting attitude had a positive effect on life skills and transfer, while a negative parenting attitude had a negative effect. Furthermore, positive and negative parenting attitudes indirectly affected life skills transfer by mediating life skills development. The findings of this study show that the role of parents determines the possibility of life skills development and transfer of Chinese student-athletes.

\section{Introduction}

In China, the student-athlete system has been implemented to foster elite sport. It has been reported that approximately 460,000 student-athletes are solely dedicated to sport (Gou, 2019). The support at the Chinese government level has established a foundation for China to obtain great results in various world competitions, including the Summer and Winter Olympics (Ling \& Hong, 2014). However, unlike the impressive superficial achievements, minor and major side effects have been generated under the surface. In particular, the win-at-all-cost philosophy in sport has hindered the balanced development of body, emotion, and psychology of student-athletes. This unbalanced physical education system has affected the lives of student-athletes adversely after their retirement. For instance, world gymnastics champion Zhang Shang Wu was arrested by the police after trying to steal due to the hardships of life after his retirement (Ling \& Hong, 2014). This incident greatly impacted not only the sport world in China but also Chinese society. This has increased a demand for support for student-athletes to achieve balanced physical and psychological development through sport.

Representatively, Various studies have been conducted in the field of sport psychology based on the positive youth development (PYD) through sport (Holt, 2016). This view sees the youth as beings with the potential and competence to create a righteous society, not as beings who have to solve developmental problems (Holt, 2016). Competence in this context is used as inner assets that influence the youth to grow positively. Among them, "life skills" are the most representative internal asset that PYD researchers have paid attention to. Holt, Deal, and Smyth (2016), Cronin and Allen (2017) argued that life skills could be used as a concrete and practical strategy to alleviate various side effects found in elite sport fields. It is because student-athletes can develop their social, psychological, and emotional strengths through sport, and as a result, balanced development and sport values can be transferred to their lives (Holt, Deal, \& Pankow, 2020). 
Life skills are defined as "skills that help the youth adapt and cope successfully in a variety of environments such as a school, home, and community" (Hodge, Danish, \& Martin, 2013). Specifically, they include various psychological, emotional, and behavioral skills such as goal setting, emotion control, problem-solving, positive thinking, and communication (Lim, Bae, \& Jang, 2018). Ultimately, they pursue transfer. Transfer refers to the ability to internalize life skills learned in sport and generalize them to life outside sport (Pierce, Gould, \& Camire, 2017). Transfer aims to help the youth have happy and valuable lives by developing life skills through sport (Weiss, Bolter, \& Kipp, 2014). However, Shek et al. (2020) reported that approximately $18 \%$ of students in China felt that their life skills were lacking. Moreover, the level of their life skills decreased as their grade was higher because school education only focused on grades and outcomes. In particular, the need for a balanced development of student-athletes began to persuade people more because of the social maladjustment of Chinese student-athletes. Currently, various life skills and transfer have been studied based on PYD.

Early life skill researchers supported the implicit approach that life skills could be developed and transferred sufficiently just by participating in sport (Jones \& Lavallee, 2009). However, recent studies support the explicit approach that it is more effective when significant others (e.g., coach, parents, peers, etc.) deliberately mediate and provide appropriate interventions (Holt et al., 2017). Gould and Carson (2008) argued that coaches and parents act as key facilitators for student-athletes in the process of developing and transferring life skills. Pierce et al. (2017) also emphasized the role of parents and coaches and included them as key factors in the life skills transfer model. In fact, Cronin and Allen (2018) reported that the autonomy support climate formed by the coach directly affected the life skills development, self-esteem, positive affect, and satisfaction with life of student-athletes.

However, as pointed out by Harwood and Knight (2015), there are far fewer studies on parental involvement compared to studies on coaches. Holt et al. (2017) conducted a meta-analysis using 63 studies on PYD and analyzed studies on significant others. The result showed that there were 19 studies on coaches, but only 9 studies were on parents. The result of this study supports the argument that studies in the PYD field have paid less attention to parents.

From a PYD perspective, parents are key elements in external assets (Holt \& Neely, 2011; Holt et al., 2017). This is because parents have the closest relationship with their children (student-athletes) biologically, socially, and psychologically. Furthermore, a parenting attitude is a very important factor influencing the positive development of student-athletes (Knight \& Holt, 2014). In general, parenting attitude can be divided into two dimensions: positive or negative (Skinner, Johson, \& Snyder, 2005). The positive parenting attitude is composed of warmth, autonomy support, and structure, while warmth indicates child-centered, affectionate, and reward-using attitudes, autonomy support means letting children make their own decisions and emphasizes responsibility for them, and structure is to provide clear expectations and consistency for mature behaviors (Holt \& Neely, 2011). On the other hand, negative parenting attitude can be classified into rejection, coercion, and chaos, where rejection indicates behaviors accusing and showing hostility to children, coercion was behaviors of excessively interfering with children without 
explaining how and why things are done, and chaos means unpredictable or ambiguous behaviors (Akcinar \& Baydar, 2014).

Dorsch, Smith, and McDonough (2015) reported that positive parental support and attitude helped student-athletes build good relationships with others and develop life skills. For example, parents can give important lessons to their children for helping them learn life skills by interacting with them behaviorally and emotionally through sport (Dorsch et al., 2015). Moreover, the praise and understanding behaviors of parents perceived by the youth positively affect the development of life skills such as teamwork, goal setting, and leadership (Mossman \& Cronin, 2019). Furthermore, the interactions between parents and sport coaches promote the development and transfer of life skills for the youth participating in sport programs (Newman, Anderson-Butcher, \& Amorose, 2020). The results of these previous studies suggest that the positive parenting attitude directly or indirectly influences the athletes to develop and transfer life skills better.

On the other hand, parenting attitudes can have a negative impact on the development of the youth, as well as a positive impact. For example, Sánchez-Miguel et al. (2013) evaluated the youth participating in elite sport to find out the relationship among parenting attitude, amotivation, and enjoyment. The results showed that the youth who perceived parenting attitude as coercion had a high level of amotivation and a low level of enjoyment. Dorsch, Smith, and Dotterer (2016) also examined student-athletes participating in team sport (i.e., American football, soccer, volleyball, etc.), and revealed that coercion and directive behaviors were positively correlated with conflict, negative affect, and ego motivation, which had a negative impact on the life skills development. In other words, negative parenting attitudes such as coercion, inconsistency, and rejection are expected to have a negative impact on the advancement of student-athletes, especially the development and transfer of life skills (Holt \& Knight, 2014).

However, negative parenting attitudes do not always affect student-athletes adversely. Mossman and Cronin (2019) studied student soccer players. The results showed that coercive and directive behaviors, commonly known as negative parenting attitudes, positively affected life skills. Newman et al. (2020) argued that life skills could be developed and transferred soundly when parenting attitudes were positive. However, some studies (Mossman \& Cronin, 2019) did not support it. O'Rourke, Smith, Smoll, and Cumming (2011) interpreted the inconsistent relationship between parenting attitude and life skills. For example, parenting attitudes such as coercion and instruction, which were perceived negatively by student-athletes, could be accepted or applied differently depending on the situation, environment, and experience. They indicated that there was no doubt that parenting attitude was an important antecedent for the life skills and transfer of student-athletes, but a more rational interpretation would be possible when more studies reflecting the characteristics of various environments, cultures, and sport would be accumulated. Mossman and Cronin (2019) also pointed out that more studies on positive or negative parenting attitudes would be needed because there were still not enough studies that statistically evaluated the relationship between parenting attitudes and life skills. 
Therefore, this study aimed to understand the relationship between the positive/negative parenting attitudes and the development and transfer of life skills by evaluating Chinese student-athletes, where people had been increasingly paying more attention to PYD recently. The research questions of this study were (a) "How does the positive parenting attitude of parents relate to sport life skills and transfer (model 1 ; paths $A 1, A 2, \& A 3)$ ?", (b) "How does the negative parenting attitude relate to sport life skills and transfer (model 2; paths B1, B2, \& B3)?", and (c) "Do sport life skills mediate the relationship between positive/negative parenting attitude and life skills transfer (paths A2' \& B2')?" The study model is shown in Fig. 1.

\section{Methods}

\section{Participants}

This study selected 270 student-athletes attending in Zhejiang, Guangdong, Jiangxi, Henan, and Shandong Provinces in China as study participants. The data of 257 participants ( 171 male and 86 female) analyzed after excluding the data of 13 participants, which were determined unfaithful or erroneous. Their mean age was 15.70 years $(S D=1.44)$. They were participating in track and field $(\mathrm{n}=54)$, basketball( $(n=68)$, soccer $(n=73)$, and taekwondo( $n=62)$.

\section{Measures}

\section{Parenting attitude}

This study used the Parents as Social Context Questionnaire for Korean adolescents (PSCQ-KA) developed by Kim and Lee (2017) to measure the parenting attitude perceived by student-athletes. The theoretical framework of this scale is the autonomy, competence, and relatedness of the theory of basic psychological needs (Skinner et al., 2005). The parenting attitude of each need can be classified according to the bipolar model. In other words, autonomy is divided into autonomy support and coercion, competence is divided into structure and chaos, and relatedness is divided into warmth and rejection. Therefore, it is composed of 24 items: four items for three positive parenting attitude factors and three negative parenting attitude factors. Items were responded using a 5-point Likert scale.

\section{Life skills}

Life skills were measured by using the Korean life skills scale for sport (KLSSS), which was verified by Lim, Kwon, Yang, Yun, and Bae (2019) for student-athletes. The questionnaire measures the life skills obtained by student-athletes in a sport environment. The KLSSS consists of 18 items, including five factors (i.e., goal setting, teamwork, interpersonal and social skills, time management, and leadership). Items were responded using a 5-point Likert scale.

\section{Life skills transfer}


Life skill transfer was measured by the Korean life skills transfer survey (KLSTS) validated by Lim et al. (2018). This test measures whether life skills learned in a sport environment are transferred and used in a non-sport environment. The scale consists of 38 items with 8 factors: helping each other, making healthy choice, appreciating diversity, meeting and greeting, goal setting, resolving conflict with friends, resolving conflict with siblings, and managing emotion. Items were responded using a 5-point Likert scale.

\section{Translation of measures}

The utilized measurement was translated from Korean to Chinese. The translation procedure was as follows. Firstly, the first draft was translated by a group of experts (one professor of sport psychology, two doctors in sport psychology, and one doctor in contemporary Chinese literature). The one professor of sport psychology and two doctors in sport psychology reviewed the contents of the Korean version measurement tool, and a bilingual speaker (the doctor in contemporary Chinese literature) who is fluent in Chinese and Korean translated it.

Secondly, the items translated into Chinese were consulted with a Chinese expert with experience in developing items and parts that were difficult to understand or inappropriate parts were modified. For example, the direct translation of "I can get along with children from multicultural families" was revised to "I can get along well with children from ethnic minority families" to reflect Chinese culture. Chinese nation refers to all 56 ethnic groups. The Chinese have many opportunities to meet friends from ethnic minority families.

The revised items were reviewed again by two bilingual users. They tested content validity through the translation-backtranslation process that translated the items translated into Chinese back to Korean and compared it with the original version. In this process, the parts that were considerably different from the original in meaning were revised through consultation with experts including bilingual users. For example, "Ask a question to a person you meet for the first time comfortably" among life skill transfer items was backtranslated from Chinese into Korean and it was presented as "Ask a question to a person you meet for the first time." This can be understood that you should ask a question to someone you never met before, contrary to the intention of the original item (i.e., you can talk to a stranger comfortably). Therefore, this item was re-translated as "I can talk with someone I meet for the first time comfortably." After completing this process, the validity and reliability of the finally translated items were analyzed.

\section{Validity and reliability of measuring tools}

Confirmatory Factor Analysis (CFA) and reliability analysis (Cronbach' a) were conducted to secure the validity and reliability of the translated measurement tool. $\mathrm{Q}\left(\chi^{2} / d f\right), \mathrm{CFI}, \mathrm{TLI}, \mathrm{RMSEA}$, and SRMR values were used for calculating the fit of the model (Kline, 2015). It was determined as "very good fit" when a Q value was 3 or less, CFI and TLI were .9 or more, and RMSEA and SRMR were .08 or less (Kline, 2015). In addition, the statistical significance of the regression coefficient was examined to secure validity. The CFA model of parenting attitudes $(\mathrm{Q}=2.38, \mathrm{CFI}=.93, \mathrm{TLI}=.92$, RMSEA $=.07, \mathrm{SRMR}=.06)$ and life skills $(\mathrm{Q}=2.14, \mathrm{CFI}=.95, \mathrm{TLI}=.94, \mathrm{RMSEA}=.07, \mathrm{SRMR}=.04)$ showed that all fit indices were above the criterion. In 
the case of life skill transfer $(\mathrm{Q}=2.47, \mathrm{CFI}=.89, \mathrm{TLI}=.88, \mathrm{RMSEA}=.08, \mathrm{SRMR}=.05)$, although CFI and TLI values did not meet the criterion, they were interpreted as "acceptable fit" because they were approximate values and the remaining three fit indices satisfied the criterion (Kline, 2015). Moreover, the Cronbach' a of each sub-factor was between .79 and .94 .

\section{Procedure}

This study received research ethics approval from the Institutional Review Board (IRB). The researcher contacted the officials of Chinese physical education middle and high schools in advance for seeking cooperation to select study participants. The researcher also sent materials including the research participant recruitment announcement and consent form via e-mail to athletes and their parents through these officials. A link containing an online questionnaire was also attached at the same time. However, it was emphasized that participation could not be forced by coaches or team officials if athletes and parents did not want to participate in the study. In addition, the anonymity and intended use of the collected data were clearly explained. Online data collection was conducted from December 2020 to January 2021. The collected data were automatically coded, and the researcher downloaded the coded data online and used them for analysis.

\section{Data analysis}

This study used SPSS version 25 and AMOS version 25, statistical packages, to analyze the data. The analysis methods are as follows. First, among the collected data, data judged to be unfaithful or erroneous were excluded in a data cleaning process. Second, this study conducted descriptive statistics to grasp the general trend of the data and to test normality. Mean, standard deviation, skewness, and kurtosis were calculated for descriptive statistics. For skewness and kurtosis, the criterion of skewness was an absolute value of 3 or less, and that of kurtosis was an absolute value of 8 or less, which were standard values implying a normal distribution (Kline, 2015). Third, Pearson's $r$ was used to examine the correlation between the sub-factors constituting these three variables. Fourth, to evaluate the study questions, the structural model was tested using AMOS. Q $\left(\chi^{2} / d f\right), \mathrm{CFI}, \mathrm{TLI}, \mathrm{RMSEA}$, and SRMR were used as fit indices as suggested by Kline (2015). After that, the path coefficient was examined, and bootstrapping was used to test mediating (indirect) effects. The number of sampling with replacement for bootstrapping was set to 2,000, and significance was determined using the two-tailed significance at $95 \%$ bias-corrected confidence interval. If 0 was not included in the derived confidence interval, the null hypothesis was rejected and the mediating effect was determined significant (Kline, 2015). All statistical significance was determined with a p-value less than .05.

\section{Results}

\section{Descriptive statistics and correlation}


The results of this study showed that the mean ranged between 2.10 and 3.85 , and the standard deviation ranged between .68 and to .89 . Skewness ranged from -.39 to .88 , and kurtosis ranged from -.63 to .85 . Considering the criteria of skewness and kurtosis (skewness $<3$, and kurtosis $<8$ ), it was determined that the data satisfied all normality assumptions (Kline, 2015). Table 1 shows the correlation analysis results between each sub-factor. It was confirmed that all sub-factors of the three variables were significantly correlated. In particular, the three factors (warmth, autonomy, and structure) included in the positive parenting attitude were positively correlated with all life skill and transfer factors, while the negative parenting attitude factors (rejection, coercion, and chaos) were negatively correlated with them.

Table 1. Correlation between all measures

\begin{tabular}{|c|c|c|c|c|c|c|c|c|c|c|c|c|c|c|c|c|c|c|c|}
\hline 1. warmth & 1 & & & & & & & & & & & & & & & & & & \\
\hline 2. autonomy & $.68^{* *}$ & 1 & & & & & & & & & & & & & & & & & \\
\hline 3. structure & $.59^{* *}$ & $.69^{* *}$ & 1 & & & & & & & & & & & & & & & & \\
\hline 4. rejection & $-.32^{* *}$ & $-.48^{* *}$ & $-.33^{*+}$ & 1 & & & & & & & & & & & & & & & \\
\hline 5. coercion & $-.35^{+*}$ & $-.50^{4 *}$ & $-.43^{*+}$ & $.61^{* 4}$ & 1 & & & & & & & & & & & & & & \\
\hline 6. chaos & $-.31^{* *}$ & $-.43^{*+}$ & $-.37^{*+}$ & $.56^{4 *}$ & $.70^{* *}$ & 1 & & & & & & & & & & & & & \\
\hline 7. TW & $.45^{\circ}$ & $.32^{* *}$ & $.37^{* *}$ & $-.18^{*+}$ & $-.18^{*+}$ & $-.26^{*+}$ & * 1 & & & & & & & & & & & & \\
\hline 8. GS & $.48^{* *}$ & $.40^{\circ *}$ & $.43^{4 *}$ & $-.17^{* *}$ & $-.18^{* *}$ & $-.20^{+*}$ & $.65^{* 4}$ & 1 & & & & & & & & & & & \\
\hline 9. TM & $.46^{\circ *}$ & $.41^{* *}$ & $.42^{* *}$ & $-.16^{*+}$ & $-.22^{+*}$ & $-.28^{*+}$ & $.54^{* *}$ & $.76^{64}$ & 1 & & & & & & & & & & \\
\hline 10. SS & $.42^{* *}$ & $.35^{4 *}$ & $.36^{4 *}$ & $-.19^{* *}$ & $-.14^{*}$ & $-.21^{*+}$ & $.60^{* 4}$ & $.59^{* 4}$ & $.53^{*+}$ & * 1 & & & & & & & & & \\
\hline 11. LD & $.42^{* *}$ & $.43^{\circ *}$ & $.44^{* *}$ & $-.13^{*}$ & $-.22^{* *}$ & $-.21^{*+}$ & $.65^{* 4}$ & $.69^{* 4}$ & $.64^{*+}$ & $.73^{* *}$ & 1 & & & & & & & & \\
\hline 12. MG & $.43^{* *}$ & $.43^{* *}$ & $.43^{* *}$ & $-.18^{* *}$ & $-.16^{*}$ & $-.23^{* *}$ & $.53^{* *}$ & $.47^{* *}$ & $.43^{* *}$ & $.65^{* *}$ & $.62^{* *}$ & 1 & & & & & & & \\
\hline 13. ME & $.41^{* *}$ & $.42^{* *}$ & $.44^{* *}$ & $-.26^{+*}$ & $-.25^{* *}$ & $-.32^{*+}$ & $.53^{* *}$ & $.47^{\circ *}$ & $.42^{* *}$ & $.54^{* *}$ & $.52^{* *}$ & $.68^{\circ *}$ & 1 & & & & & & \\
\hline 14. GS_T & $.47^{* *}$ & $.50^{* *}$ & $.54^{* *}$ & $-.22^{*+}$ & $-.28^{*+}$ & $-.36^{+4}$ & $.57^{* *}$ & $.63^{*+}$ & $.60^{* *}$ & $.53^{* *}$ & $.63^{* *}$ & $.63^{* *}$ & $.71^{* *}$ & 1 & & & & & \\
\hline 15. RF & $.46^{* 4}$ & $.50^{\circ *}$ & $.56^{* *}$ & $-.22^{*+}$ & $-.21^{* *}$ & $-.32^{*+}$ & $+.59^{* *}$ & $.55^{\circ+}$ & $.54^{* *}$ & * $.60^{* *}$ & $.63^{4 *}$ & $.69^{* 4}$ & $.68^{4 *}$ & $.75^{* *}$ & 1 & & & & \\
\hline 16. RS & $.47^{* *}$ & $.47^{* *}$ & $.56^{4 *}$ & $-.23^{* *}$ & $-.27^{+*}$ & $-.33^{*+}$ & $.53^{* *}$ & $.52^{* *}$ & $.55^{\circ+}$ & $.53^{* *}$ & $.60^{4 *}$ & $.61^{* *}$ & $.63^{4 *}$ & $.71^{* *}$ & $.82^{* *}$ & 1 & & & \\
\hline 17. MH & $.50^{* *}$ & $.50^{\circ *}$ & $.50^{* *}$ & $-.22^{* *}$ & $-.27^{+*}$ & $-.34^{*+}$ & $.56^{* *}$ & $.64^{* 4}$ & $.61^{* *}$ & $.56^{\circ *}$ & $.58^{* *}$ & $.63^{* *}$ & $.61^{* *}$ & $.70^{* *}$ & $.74^{* *}$ & $.71^{* *}$ & 1 & & \\
\hline 18. $\mathrm{AD}$ & $.44^{* *}$ & $.35^{* *}$ & $.33^{* *}$ & $-.27^{* *}$ & $-.16^{+}$ & $-.24^{*+}$ & $+.59^{* *}$ & $.54^{* 4}$ & $.49^{* *}$ & * $.58^{* *}$ & $.51^{* *}$ & $.62^{* 4}$ & $.69^{* *}$ & $.68^{* *}$ & $.68^{* *}$ & $.62^{* *}$ & $.65^{* *}$ & 1 & \\
\hline 19. $\mathrm{HE}$ & $.50^{* *}$ & $.43^{\circ *}$ & $.50^{4 *}$ & $-.24^{*+}$ & $-.23^{*+}$ & $-.31^{*+}$ & $.57^{* *}$ & $.57^{* *}$ & $.51^{* 4}$ & * $.63^{* *}$ & $.54^{* *}$ & $.64^{*+}$ & $.63^{4 *}$ & $.68^{* 4}$ & $.74^{* *}$ & $.65^{* *}$ & $.71^{* *}$ & $.69^{* *}$ & 1 \\
\hline $\mathrm{M}$ & 3.63 & 3.83 & 3.64 & 2.10 & 2.58 & 2.60 & 3.71 & 3.66 & 3.37 & $7 \quad 3.54$ & 3.19 & 3.49 & 3.75 & 3.66 & 3.71 & 3.71 & 3.66 & 3.85 & 3.82 \\
\hline SD & .83 & .83 & .86 & .84 & .89 & .77 & .70 & .68 & .80 & .77 & .83 & .79 & .73 & .72 & .72 & .72 & .70 & .75 & .69 \\
\hline
\end{tabular}

${ }^{*} p<.05,{ }^{* *} p<.01$

Note. $\mathrm{TW}=$ teamwork, $\mathrm{GS}=$ goal setting, $\mathrm{TM}=$ time management, $\mathrm{SS}=$ social skill, $\mathrm{LD}=$ leadership, $\mathrm{MG}=$ Meeting and Greeting, ME= Managing Emotion, GS_T=Goal Setting of Transfer, RF=Resolving Conflict with Friends, $\mathrm{RS}=$ Resolving Conflict with Sibling, $\mathrm{MH}=$ Managing Healthy Choice, $\mathrm{AD}=$ Appreciating Diversity, $\mathrm{HE}=$ Helping Each 


\section{Structural models}

Model 1: Relationship among positive parenting attitude, life skills, and transfer

The fit indices of the model 1 were $\mathrm{Q}=2.65, \mathrm{CFI}=.95, \mathrm{TLI}=.94, \mathrm{RMSEA}=.08$, and SRMR $=.04$. Since all values met the criteria of "very good fit", it was determined that they were suitable for this model (Kline, 2015). The regression coefficient for each path is presented in Table 2. First, a positive parenting attitude positively affected sport life skills $(\beta=.61, p<.001)$. Second, a positive parenting attitude positively influenced life skill transfer $(\beta=.27, p<.001)$. Third, sport life skills had a positive effect on life skill transfer $(\beta=.68, p<.001)$.

Table 2. Relationship among positive parenting attitude, sport life skills, and transfer

\begin{tabular}{lcccc} 
Path & B & $\beta$ & S.E. & $t$ \\
\hline A1. Positive parenting attitude $\rightarrow$ Sport life skills & .51 & .61 & .06 & $8.23^{\star \star \star}$ \\
\hline A2. Positive parenting attitude $\rightarrow$ Transfer & .26 & .27 & .06 & $4.66^{\star \star \star}$ \\
\hline A3. Sport life skills $\rightarrow$ Transfer & .77 & .68 & .08 & $9.35^{\star \star \star}$ \\
\hline${ }^{* \star \star} p<.001$ & & & &
\end{tabular}

Model 2: Relationship among negative parenting attitude, life skills, and transfer

The fit indices of the model 2 were $\mathrm{Q}=2.40, \mathrm{CFI}=.96, \mathrm{TLI}=.95, \mathrm{RMSEA}=.07$, and SRMR $=.04$. Since all values satisfied the criteria of "very good fit", this model was judged to be suitable (Kline, 2015). The regression coefficient of each path is shown in Table 3. First, a negative parenting attitude had a negative impact on sport life skills $(\beta=-.30, p<.001)$. Second, a negative parenting attitude affected life skill transfer $(\beta=-.15$, $p<.01)$. Third, sport life skills influenced life skill transfer negatively $(\beta=.81, p<.001)$. The results of the model 1 and 2 are presented in Fig. 2.

Table 3. Relationship among negative parenting attitude, sport life skills, and transfer

\begin{tabular}{lcccc} 
Path & B & $\beta$ & S.E. & $t$ \\
\hline B1. Negative parenting attitude $\rightarrow$ Sport life skills & -.27 & -.30 & .07 & $-4.03^{\star \star \star}$ \\
\hline B2. Negative parenting attitude $\rightarrow$ Transfer & -.16 & -.15 & .05 & $-3.12^{\star \star}$ \\
\hline B3. Sport life skills $\rightarrow$ Transfer & .91 & .81 & .08 & $11.15^{\star \star \star}$ \\
\hline${ }^{* \star *} p<.001$ & & & &
\end{tabular}




\section{Testing mediation effects}

The lower bound of positive parenting attitude's confidence intervals was .28 and the upper bound of it was .55. Based on these values, the mediating effect was determined significant. Therefore, it was concluded that the positive parenting attitude perceived by student-athletes affected the transfer of life skills positively through sport life skills $(\beta=.42, p<.001)$. On the other hand, the lower bound of negative parenting attitude's confidence intervals was -.44 and the upper bound of it was -.08 . It was judged to be significant based on these values. Therefore, it was concluded that the negative parenting attitude perceived by student-athletes influenced the transfer negatively through sport life skills $(\beta=-.24, p<.01)$.

\section{Discussion}

The objective of this study was to examine the structural models of positive/negative parenting attitude, sport life skill, and transfer targeting Chinese student-athletes. This study collected data from 257 middle and high school students to achieve the objective. This study analyzed the collected data using descriptive statistics, correlation analysis, and structural equation modeling.

\section{Model 1: Relationship among positive parenting attitude, life skills, and transfer}

The model 1 showed that positive parenting attitudes positively affected life skills and transfer. Life skills also affected transfer positively. The results indicated that if parented supported autonomy, provided a coherent structure, and showed affectionate attitudes, it could help student-athletes develop and transfer life skills. These results agreed with the results of various previous studies (Gould \& Carson, 2008; Holt et al., 2017) that claimed the relationship between positive parenting behaviors and life skills. Yun, Lim, and Jang (2017), who reported that parenting attitudes influenced the character and morality of athletes, and Mossman and Cronin (2019), who argued that positive parenting attitudes affected the life skills and enjoyment of student-athletes, also supported the results of this study.

Hodge et al. (2013) proposed the LDI/BNT LS (conceptual model of LS development) model. This model explains the relationship between parenting attitudes and the life skills of student-athletes in connection with basic psychological needs. This model argues that the basic psychological needs and supportive climate formed under the influence of significant others play a critical role in the development and transfer of life skills for the youth participating in sport. In other words, student-athletes satisfy their basic psychological needs by perceiving positive parenting attitudes, and they learn higher self-initiative and learning motivation through this. Moreover, a high level of intrinsic motivation helps them positively interact with other sport participants (Wheeler \& Green, 2014). In fact, Johnston, Harwood, and Minniti (2013) quantitatively proved that student-athletes with high participation motivation were more advantageous in developing life skills such as goal setting, time management, and problem-solving. Therefore, the positive attitude of parents rearing them is important for student-athletes to develop life skills in sport and use them in their lives. 
Several studies have reported that intervention programs improved parenting attitudes. For example, Juffer, Bakermans-Kranenburg, and van IJzendoorn (2017) studied the promotion of parenting attitudes and reported that the Video-feedback Intervention to promote Positive Parenting and Sensitive Discipline (VIPP-SD) increased sensitive caregiving, improved parental self-efficacy, and provided more favorable attitudes toward caregiving and limit setting. Spencer, Topham, and King (2020) meta-analyzed 28 studies and also reported that online education programs for parents helped the promotion of positive parenting attitudes.

However, as indicated earlier, even though interest in significant others and the development of life skills for the youth have been increasing in the sport PYD field, still relatively little interest has been given to the role of parents. In fact, many researchers (Camiré, Kendellen, Rathwell, \& Turgeon, 2020; Turgeon, Camiré, \& Rathwell, 2021) have evaluated PYD online education for coaches, but there is no content on parent education. As shown by the results of this study, a positive parenting attitude, indicating the active support and encouragement of parents, is most important. Therefore, it is necessary to develop and provide intervention means for parents of children who participate in sport to acquire useful information.

\section{Model 2: Relationship among negative parenting attitude, life skills, and transfer}

Negative parenting attitudes affected sport life skills and transfer negatively. These results agreed with the argument of many researchers (e.g., Gould \& Carson, 2008; Holt et al., 2017) that the development of the youth participating in sport could vary depending on the parenting attitudes of parents toward their children. Danioni, Barni, and Rosnati (2017) reported that parental pressure reduces positive psychological and emotional variables (e.g., the competence, self-esteem, and pleasure of the youth) that could be experienced in sport. It was also supported by the argument of Skinner et al. (2005) that the rejecting attitude of parents weakened the relatedness of children, inconsistency hindered the formation of children's competence experience, and coercion interfered with the psychological autonomy development of children.

One cause of this result could be the collectivism atmosphere in China. Chinese parents have a higher level of interest and involvement because they emphasize the college admission of their children more than the parents in the western culture (individualism) (Fong, 2007). It is because Chinese parents have a strong tendency to consider their children's success as a family glory. Yoshikawa, Way, and Chen (2012) analyzed the Chinese parents and found that parents' anxiety about their children's academic performance increased as their children advanced through school. Moreover, parents who feel anxious about their children's studies are more likely to show rejection, coercion, and inconsistent negative parenting attitudes toward their young children (14-18 years old). In other words, parents of studentathletes would force their children to win in games so that they can go to good colleges. A number of studies have proved that this negative parenting attitude results in the negative developmental outcomes of student-athletes such as aggression, antisocial behaviors, stress, and negative emotions (Dorsch et al., 2016). 
On the other hand, it is necessary to understand that the cultural background of China is rooted in Confucianism. In oriental cultures, vertical relationships according to age and position are implicitly applied. It can be also found in the parent-child relationship. Parents who raise children based on Confucian values tend to demand their children to behave as they want while keeping a certain distance from their children because they believe that they should show prestige as parents (Chuang, Glozman, Green, \& Rasmi, 2018). In this home environment, student-athletes are more likely to do actions (e.g., excessive social comparison, and anti-social behavior) that impede their positive development without hesitation to receive attention and recognition from their parents.

In general, negative parenting attitudes tend to make student-athletes self-goal-oriented and intoxicated with the win-at-all-cost philosophy (Keegan, Harwood, Spray, \& Lavallee, 2009). Under the atmosphere, student-athletes are more likely to experience negative development, as opposed to PYD, in order to improve their match performance or to meet their parents' needs or expectations. In particular, negative parenting attitudes adversely affect children's mental health such as depression as well as cause the negative development of children (Bruce et al., 2006). Christofferson and Strand (2016) argued that it is necessary for parents to (a) understand the philosophical values and structure of sport, (b) prevent misunderstanding with referees and coaches by clearly understand the rules of sport, (c) create a conflictfree environment by understanding sportpersonship, and (d) know how to present positive behaviors by recognizing and regulating their emotions in order to improve negative parenting attitude.

\section{Testing mediation effects}

Positive and negative parenting attitudes had positive and negative impacts on life skill transfer, respectively, through sport life skills. The results showed that parenting attitudes affected the transfer of life skills indirectly as well as directly. The result of this study concurred with the results of previous studies (Newman et al., 2020) revealing that the role of parents was important for promoting the positive development of the youth.

Pierce et al. (2017) did not view that the transfer of life skills always produced positive results. For example, attacking the opponent's weaknesses is a good strategy for victory in various sport such as soccer and taekwondo, but it is not always true in daily life. In the long run, it is rather likely to be socially criticized to achieve success by taking advantage of the other person's weakness in life. Therefore, it should be remembered that knowing the true meaning and use of life skills in a sport environment and put them into practice can be positive or negative depending on the role of their parents.

It is commonly accepted that the behavior and language of parents are key variables greatly influencing the development of children (Mossman \& Cronin, 2019; Ramachandran, 2000). In particular, modeling presented by the social learning theory specifically explains how parental behavior can affect the transfer of student-athletes' life skills (Bandura, 1991; Kerr et al., 2019). According to this theory, student-athletes imitate what they see and feel consciously or unconsciously. For example, student-athletes who see the inconsistent behavior of their parents (e.g., not keeping promises, not complying with rules, and changing behavior depending on their mood) are more likely to show a similar tendency. On the other hand, children 
of parents who set and implement clear rules and plans can learn elements (e.g., time management and goal setting) that help them develop life skills. In other words, the successful transfer of student-athletes' life skills is directly or indirectly influenced by various parenting behaviors that are provided by their parents in daily life, such as opportunities to use the skills, support, rewards, and detailed explanations.

The relationship between positive/negative parenting attitudes and the development and transfer of student-athletes' life skills can also be found in neuroscience. In particular, the mirror neuron system theory suggests that parents can be the behavior mirror of their children (Oberman, Pineda, \& Ramachandran, 2007). This is supported by the fact that the brain part used while acting and that activated when observing or imagining something are identical (Sternberg, 2016). For instance, when student-athletes observe their parents' behavior, the same brain part activated while acting is stimulated. As this stimulus accumulates, the possibility of a behavior increases. However, their brains are not yet able to determine whether the behavior of their parents is right or wrong. Regardless of the value of the observed parental behavior, the brain operates upon a command. Therefore, parents always need to examine their own actions and the consequences of them.

\section{Future direction}

First, although this study focused on the role of parents, the influence of coaches cannot be ignored in the sport environment (Bae, Lim, \& Jang, 2019; Pierce et al., 2017). In particular, it is necessary to pay attention to the interaction effect of the two groups rather than the individual influence of parents or coaches. As Newman et al. (2020) pointed out, parents and coaches influence the development and transfer of student-athletes' (or youths participating in sport) life skills separately, but the level of outcomes can vary depending on how the two groups interact. For example, the development and transfer of life skills of a student-athlete who has a democratic coach and coercive parents can be different from those of a student-athlete who has a democratic coach and affectionate parents. Therefore, future studies need to evaluate the roles of parents and coaches comprehensively rather than examining a parent group and a coach group separately.

Second, it is necessary to develop a tool that can measure whether parents teach life skills in order to more specifically test the influence of parents on the development and transfer of student-athletes' life skills. Recently, a scale was developed to evaluate the life-skill coaching level of coaches who are the significant others in the sport field (Camiré et al., 2021). Researchers can evaluate the effects of the lifeskill coaching level of coaches on the development and transfer of student-athletes' life skills by using this scale. This supports the explicit approach that the largest effect can be acquired when teaching life skills intentionally (Lim, 2019). However, there is no scale for measuring the life-skill coaching of parents. Therefore, future studies are needed to develop measuring tools that can evaluate whether parents intentionally teach life skills to their children.

Third, it should be noted that student-athletes and parents interact. Interaction means that two parties affect each other. It is highly likely that student-athletes and their parents are more likely to interact with each other while developing and transferring life skills. The Actor-Partner Independence Model (APIM) 
can be used to statistically test this. APIM is a statistical technique commonly used to examine the relationship between variables that interact between a husband and a wife or between parents and a child. Therefore, future studies may employ different methodological approaches such as APIM to objectively evaluate the interaction between student-athletes and parents.

\section{Conclusion}

The following conclusions were drawn according to a series of results. First, a positive parenting attitude affects the development and transfer of student-athletes' life skills positively. Second, a negative parenting attitude negatively influences the development and transfer of student-athletes' life skills. Third, a positive parenting attitude has a positive and indirect effect on transfer through sport life skills. On the other hand, a negative parenting attitude has a negative and indirect impact on transfer through sport life skills.

In conclusion, a positive parenting attitude helps student-athletes develop life skills, and life skills are transferred to their lives positively. On the other hand, a negative parenting attitude hinders the development of student athletes' life skills, and life skills are transferred negatively to life. Moreover, life skills act positively or negatively between parenting attitudes and transfer.

\section{Declarations}

Data Availability Statement: The data presented in this study are available on request from the corresponding author. The data are not publicly available due to privacy issues.

Informed Consent Statement: Informed consent was obtained from all the parents/ legal guardians of all the participants involved in the study.

Conflicts of Interest: The authors declare no conflicts of interest.

Funding: This research received no external funding.

Institutional Review Board Statement: The study was conducted in accordance with the Declaration of Helsinki, and the protocol was approved by the Institutional Review Board (IRB) of Yongin University (IRB No. 2-1040966-AB-N-01-2102-HSR-2110).

Author contributions: Q. Y. W. and T. L. conceptualization; Q. Y. W. and J. B. formal analysis; T. L. methodology; Q. Y. W. project administration; T. L. supervision; T. L. and J. B. validation; Q. Y. W. and J. B. writing-original draft, T. L. writing- review \& editing; All authors reviewed the manuscript.

\section{References}

1. Akcinar, B., \& Baydar, N. (2014). Parental control is not unconditionally detrimental for externalizing behaviors in early childhood. International Journal of Behavioral Development, 38(2), 118-127. 
2. Bae, J. S., Lim, T. H., \& Jang, C. Y. (2019). Exploring the life skills model for Taekwondo education. Korean Journal of Sport Psychology, 30(1), 81-91

3. Bandura, A. (1991). Social cognitive theory of self-regulation. Organizational behavior and human decision processes, 50(2), 248-287.

4. Bruce, A. E., Cole, D. A., Dallaire, D. H., Jacquez, F. M., Pineda, A. Q., \& LaGrange, B. (2006). Relations of parenting and negative life events to cognitive diatheses for depression in children. Journal of abnormal child psychology, 34(3), 310-322.

5. Camiré, M., Kendellen, K., Rathwell, S., \& Turgeon, S. (2020). Evaluating the Coaching for Life Skills online training program: A randomised controlled trial. Psychology of Sport and Exercise, 48, 101649.

6. Camiré, M., Turgeon, S., Kramers, S., Rathwell, S., Bean, C., Sabourin, C., \& Pierce, S. (2021). Development and initial validation of the coaching life skills in sport questionnaire. Psychology of Sport and Exercise, 53, 101845.

7. Chuang, S. S., Glozman, J., Green, D. S., \& Rasmi, S. (2018). Parenting and family relationships in Chinese families: A critical ecological approach. Journal of Family Theory \& Review, 10(2), 367-383.

8. Cronin, L. D., \& Allen, J. (2017). Development and initial validation of the Life Skills Scale for Sport. Psychology of Sport and Exercise, 28, 105-119.

9. Cronin, L. D., \& Allen, J. (2018). Examining the relationships among the coaching climate, life skills development and well-being in sport. International journal of sports science \& coaching, 13(6), 815827.

10. Danioni, F., Barni, D., \& Rosnati, R. (2017). Transmitting sport values: The importance of parental involvement in children's sport activity. Europe's journal of psychology, 13(1), 75-92.

11. Dorsch, T. E., Smith, A. L., \& Dotterer, A. M. (2016). Individual, relationship, and context factors associated with parent support and pressure in organized youth sport. Psychology of Sport and Exercise, 23, 132-141.

12. Dorsch, T. E., Smith, A. L., \& McDonough, M. H. (2015). Early socialization of parents through organized youth sport. Sport, exercise, and performance psychology, 4(1), 3-18.

13. Fong, V. L. (2007). Morality, cosmopolitanism, or academic attainment? Discourses on "quality" and urban Chinese-only-children's claims to ideal personhood. City \& Society, 19(1), 86-113.

14. Gou, Z.W. (2019). 70 Years of Sports in New China. China Sports News, 001. DOI: 10.38308/n.cnki.nzgty.2019.001508

15. Gould, D., \& Carson, S. (2008). Life skills development through sport: Current status and future directions. International review of sport and exercise psychology, 1(1), 58-78.

16. Harwood, C. G., \& Knight, C. J. (2015). Parenting in youth sport: A position paper on parenting expertise. Psychology of sport and exercise, 16, 24-35.

17. Hodge, K., Danish, S., \& Martin, J. (2013). Developing a conceptual framework for life skills interventions. The Counseling Psychologist, 41(8), 1125-1152.

18. Holt, N. L. (Ed.). (2016). Positive youth development through sport. Routledge. 
19. Holt, N. L., \& Knight, C. J. (2014). Parenting in youth sport: From research to practice. Routledge.

20. Holt, N. L., \& Neely, K. C. (2011). Positive youth development through sport: A review. Revista de Iberoamericana de Psicologia del Ejercicio y el Deporte, 6(2), 299-316

21. Holt, N. L., Deal, C. J., \& Pankow, K. (2020). Positive youth development through sport. Handbook of Sport Psychology, 429-446.

22. Holt, N. L., Deal, C. J., \& Smyth, C. L. (2016). Future directions for positive youth development through sport. Positive youth development through sport, 229-240.

23. Holt, N. L., Neely, K. C., Slater, L. G., Camiré, M., Côté, J., Fraser-Thomas, J., ... \& Tamminen, K. A. (2017). A grounded theory of positive youth development through sport based on results from a qualitative meta-study. International review of sport and exercise psychology, 10(1), 1-49.

24. Johnston, J., Harwood, C., \& Minniti, A. M. (2013). Positive youth development in swimming: Clarification and consensus of key psychosocial assets. Journal of applied sport psychology, 25(4), 392-411.

25. Jones, M. I., \& Lavallee, D. (2009). Exploring the life skills needs of British adolescent athletes. Psychology of sport and Exercise, 10(1), 159-167.

26. Juffer, F., Bakermans-Kranenburg, M. J., \& van IJzendoorn, M. H. (2017). Pairing attachment theory and social learning theory in video-feedback intervention to promote positive parenting. Current Opinion in Psychology, 15, 189-194.

27. Keegan, R. J., Harwood, C. G., Spray, C. M., \& Lavallee, D. E. (2009). A qualitative investigation exploring the motivational climate in early career sports participants: Coach, parent and peer influences on sport motivation. Psychology of sport and exercise, 10(3), 361-372.

28. Kerr, K. L., Ratliff, E. L., Cosgrove, K. T., Bodurka, J., Morris, A. S., \& Simmons, W. K. (2019). Parental influences on neural mechanisms underlying emotion regulation. Trends in neuroscience and education, 16, 100118.

29. Kim, T., \& Lee, E. (2017). Validation of the Korean version of parents as social context questionnaire for adolescents: PSCQ_KA. Korean Journal of Youth Studies, 24(3), 313-333.

30. Kline, R. B. (2015). Principles and practice of structural equation modeling. Guilford publications.

31. Knight, C. J., \& Holt, N. L. (2014). Parenting in youth tennis: Understanding and enhancing children's experiences. Psychology of Sport and exercise, 15(2), 155-164.

32. Lim, T. H. (2019). Changes in Life Skills and Learning Attitudes of Student Athletes by Applying PEAK Program. Korean Journal of Sport Psychology, 30(2), 15-28.

33. Lim, T. H., Bae, J. S., \& Jang, C. Y. (2018). The Validation of Korean Life Skills Transfer Survey (KLSTS). Korean Journal of Sport Psychology, 29(4), 1-12.

34. Lim, T. H., Kwon, O. J., Yang, Y. K., Yun, M. S., \& Bae, J. S. (2019). Validation of the Korean Life Skills Scale for Sport (KLSSS). Korean Journal of Sport Science, 30(1), 20-33.

35. Ling, Z., \& Hong, F. (2014). After the Glory: Elite Athletes' Re-Employment in China. The International Journal of the History of Sport, 31(6), 635-651. 
36. Mossman, G. J., \& Cronin, L. D. (2019). Life skills development and enjoyment in youth soccer: The importance of parental behaviours. Journal of Sports Sciences, 37(8), 850-856.

37. Newman, T. J., Anderson-Butcher, D., \& Amorose, A. J. (2020). Examining the influence of sport program staff and parent/caregiver support on youth outcomes. Applied Developmental Science, 24(3), 263-278.

38. O'Rourke, D. J., Smith, R. E., Smoll, F. L., \& Cumming, S. P. (2011). Trait anxiety in young athletes as a function of parental pressure and motivational climate: is parental pressure always harmful? Journal of applied sport psychology, 23(4), 398-412.

39. Oberman, L. M., Pineda, J. A., \& Ramachandran, V. S. (2007). The human mirror neuron system: a link between action observation and social skills. Social cognitive and affective neuroscience, 2(1), 6266.

40. Pierce, S., Gould, D., \& Camiré, M. (2017). Definition and model of life skills transfer. International Review of Sport and Exercise Psychology, 10(1), 186-211.

41. Ramachandran, V. S. (2000). Mirror neurons and imitation learning as the driving force behind "the great leap forward" in human evolution. Retrieved March 20, 2021, from https://www.edge.org/3rd_culture/ramachandran/ramachandran_index.html

42. Sánchez-Miguel, P. A., Leo, F. M., Sánchez-Oliva, D., Amado, D., \& García-Calvo, T. (2013). The importance of parents' behavior in their children's enjoyment and amotivation in sports. Journal of human kinetics, 36(1), 169-177.

43. Shek, D. T., Lin, L., Ma, C. M., Yu, L., Leung, J. T., Wu, F. K., ... \& Dou, D. (2020). Perceptions of adolescents, teachers and parents of life skills education and life skills in high school students in Hong Kong. Applied Research in Quality of Life, 1-14.

44. Skinner, E., Johnson, S., Snyder, T. (2005). Six dimensions of parenting: a motivational model. Parenting: Science and Practice, 5(2), 175-235.

45. Spencer, C. M., Topham, G. L., \& King, E. L. (2020). Do online parenting programs create change?: A meta-analysis. Journal of Family Psychology, 34(3), 364-374.

46. Sternberg, E. (2016). NeuroLogic: The Brain's Hidden Rationale Behind Our Irrational Behavior. Vintage.

47. Turgeon, S., Camiré, M., \& Rathwell, S. (2021). Follow-up evaluation of the Coaching for Life Skills online training program. International Journal of Sports Science \& Coaching, 16(1), 173-180.

48. Weiss, M. R., Bolter, N. D., \& Kipp, L. E. (2014). Assessing impact of physical activity-based youth development programs: Validation of the Life Skills Transfer Survey (LSTS). Research quarterly for exercise and sport, 85(3), 263-278.

49. Wheeler, S., \& Green, K. (2014). Parenting in relation to children's sports participation: Generational changes and potential implications. Leisure studies, 33(3), 267-284.

50. Yoshikawa, H., Way, N., \& Chen, X. (2012). Large-scale economic change and youth development: the case of urban China. New directions for youth development, 135, 39-55. 
51. Yun, H., Lim, T., \& Jang, C. (2017). The causal Relationships of perceived Parents' Rearing Attitude on Moral Behavior in Sport among Youth Taekwondo Athletes. Korean Journal of Sport Psychology, 28(3), 61-70.

\section{Figures}
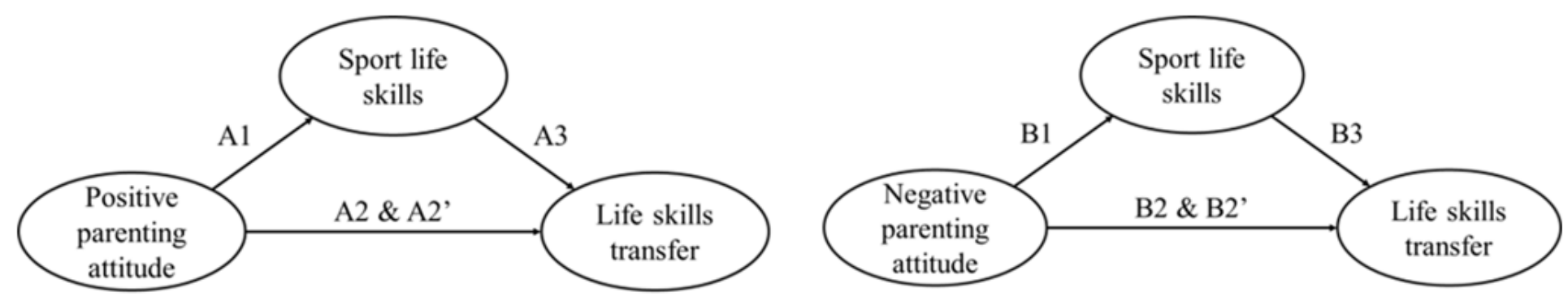

\section{Figure 1}

Research model 1 and 2 Note. Path A2' and B2' are mediation effect of sport life skills between the relationship of positive/negative parenting attitude and life skills transfer
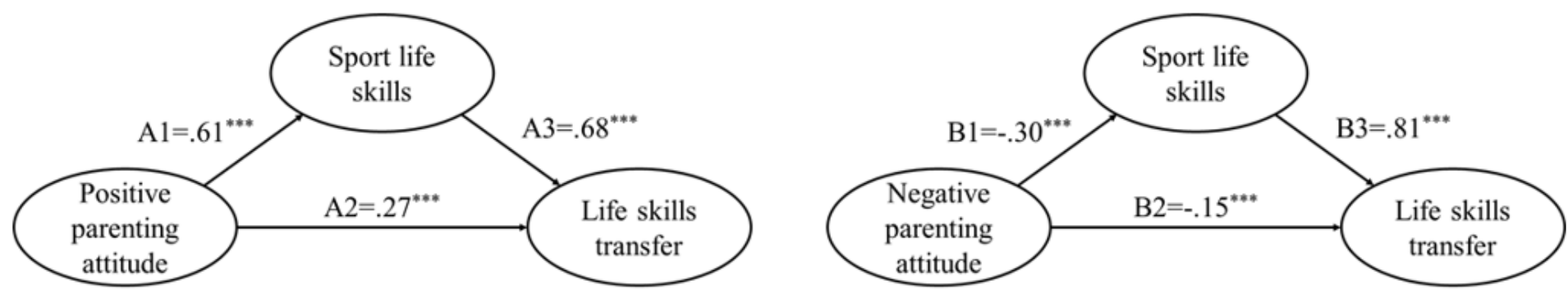

\section{Figure 2}

Research of model 1 and $2(* \star \star p<.001)$ 